\title{
Visual Outcome After Sutureless Scleral Fixation of Intraocular Lens
}

\author{
Muhammad Sajid Munir ${ }^{1}$, Muhammad Ramzan², Muhammad Arshad ${ }^{3}$, Shahid Nazir ${ }^{4}$ \\ Muhammad Rizwan Ullah ${ }^{5}$ \\ ${ }^{1-2}$ Niazi Medical and Dental College Sargodha, ${ }^{3}$ Bahawal Victoria Hospital, Bahawalpur \\ ${ }^{4}$ Red Cresent Medical and Dental College, Kasur, ${ }^{5}$ Postgraduate Medical Institute \\ Ameer-ud-Din Medical College PGMI Lahore General Hospital, Lahore
}

\begin{abstract}
Purpose: To study the results of sutureless scleral fixation of intraocular lens (IOL) in cases of complicated cataract surgery.

Study Design: Interventional case series.

Place and Duration of Study: Niazi Medical and Dental College Sargodha from Jan 2018 to Dec 2019.

Methods: Twenty-five eyes of 25 consecutive patients were selected for this study. Patients of traumatic cataract with zonular dehiscence, patients who had per-operative complications like PC rent with insufficient capsular bag, and children with subluxated lens of Marfan, Ehler Danlos syndrome were included in this study. After routine investigation all patients were operated under local anesthesia except children, who were operated under general anesthesia. Sclerotomies were made at 3 and 9 o'clock positions with MVR blade. Sutureless scleral fixation of intraocular lens was done. The haptics of lenses were buried in the scleral pockets which were already made at 3 and 9 o'clock position. Post-operative antibiotics and steroids were given for 8 weeks and patients were followed up for two years.
\end{abstract}

Results: Out of 25, $18(72 \%)$ female patients and $7(28 \%)$ male patients underwent surgery. Most of the patients had previous surgical complications of Posterior capsular rent $15(60 \%)$. Four (16\%) patients had eye trauma, three had lens subluxation for pseudo-exfoliation and 3 patients (children) were suffering from Marfan Syndrome. Seventy-two percent patients had visual acuity of more than $6 / 18,16 \%$ had $6 / 18$ to $6 / 24$ and $12 \%$ had $6 / 24$ to 6/60.

Conclusion: Properly done suture-less scleral fixation is a safe technique with little chance of IOL dislocation.

Key Words: Scleral fixation, Intraocular lens, Cataract.

How to Cite this Article: Munir MS, Ramzan M, Arshad M, Nazir S, Ullah MR. Visual Outcome after Sutureless Scleral Fixation of Intraocular Lens (IOL). Pak J Ophthalmol. 2021, 37 (1): 88-91.

Doi: https://doi.org/10.36351/pjo.v37i1.1141

Correspondence: Muhammad Ramzan

Department of Ophthalmology

Niazi Medical and Dental College

Sargodha

Email:mroph1998@gmail.com

Received: September 29, 2020

Accepted: November 15, 2020

\section{INTRODUCTION}

Cataract is the major cause of preventable blindness all over the world. Most of the cataract surgeries are uneventful and can be managed easily. In some cases, complications may occur; for example, posterior capsular rent, accidental intracapsular extraction and weak zones in pseudo exfoliation. ${ }^{1}$ We need to modify different techniques for IOL implantation in such cases. Each year approximately 18 million people suffer from ocular trauma worldwide. ${ }^{2}$ Traumatic 
cataract, lens dislocation and loss of phakic lens are the most common sequelae of ocular trauma. Marfan syndrome, Ehler Danlos syndrome, stickler syndrome where lens subluxation is common, aphakia is not a desirable long-term option.

To treat such conditions multiple options like Anterior chamber Intraocular lens (AC IOL) implantation, Iris fixed Intraocular lens and scleral fixation of IOL with 10/0 Prolene are still considered good alternatives. ${ }^{3,4}$ The AC IOL and Iris fixed IOLs are almost discouraged because of their post-operative complications. AC IOLs cause corneal decompensation and glaucoma while Iris Fixed IOLs cause chronic cystoid macular edema and Iris chafing in most patients. The risk of all these above complications are much less in cases of scleral fixation IOLs. This can be performed as either a primary or a secondary procedure. ${ }^{5,6}$ However, in case of scleral fixed IOL with 10/0 prolene suture breakage, exposure of suture knot causing inflammation and a grave complication like drop of IOL after 8 to 10 years are reported complications. ${ }^{7,8}$ In order to reduce these problems, suture-less scleral fixation is an excellent technique which has maximum advantages and minimum disadvantages. ${ }^{9}$

We carried out this case series to find out the visual and anatomical outcomes of suture-less scleral fixation of IOL in a tertiary care hospital.

\section{METHODS}

The study was conducted at Niazi Medical and Dental College Sargodha from Jan 2018 to Dec 2019. Twenty-five patients were selected on the basis of non-probability convenience sampling method. Patients of traumatic cataract with zonular dehiscence, patients of per operative complications like PC rent with insufficient capsular bag, and children with subluxated lens of Marfan, Ehler Danlos syndrome were included in this study. After routine investigation all patients were operated under local anesthesia except children, who were operated under general anesthesia.

After application of povidone iodine and draping, we marked the 3 and 9 o $^{\prime}$ clock positions. Peritomy was done and sclerotomy sites were marked $2 \mathrm{~mm}$ behind the limbus. Intra-scleral tunnels of $2 \mathrm{~mm}$ length and $50 \%$ of scleral thickness, were fashioned with 3.2 $\mathrm{mm}$ slit knife in anti-clock and clock wise movement at 3 and 9 O'clock positions respectively. An AC maintainer was inserted at anterior limbus. $25 \mathrm{G}$ MVR (Micro vitreo-retinal) blade was used to create full thickness sclerotomies parallel to the limbus at 3 and 9 O'clock. In our study we used Alcon MA 60 Model (Alcon Acrysof) intraocular lens. We made two side ports at 10 and 2'o clock position of eye. From one side port we injected intraocular lens and grasped its front haptic with 25-G IOL gripping forceps and pulled out through sclerotomy at 3 o'clock. The trailing Haptic, which was left outside was grasped with 25-G lens forceps, fed to the second instrument, inside the anterior chamber and pulled out through the sclerotomy at 9 o'clock. We buried both ends in the pockets at 3 and $90^{\circ}$ clock position. Peritomies were closed with 10/0 Nylone suture. Post-operative antibiotics and steroids were given for 8 weeks.

\section{RESULTS}

In our study most of the patients were females. Out of 25 , there were $18(72 \%)$ female patients and $7(28 \%)$ male patients. There were different indications for suture-less fixation of IOL. Most of the patients had previous surgical complications of PC rent 15 (60\%), 4 (16\%) patients had eye trauma, 3 patients had lens subluxation caused by pseudo-exfoliation and 3 patients who were children had Marfan Syndrome. The complications related with this procedure are shown in table 1 . Most of the patients achieved good visual acuity according to WHO criteria as shown in table 2 .

\section{Table 1:}

\begin{tabular}{lcr}
\hline Early Complications & No of Patients & \% \\
\hline Transient corneal edema & 5 & 20 \\
Mild vitreous hemorrhage & 4 & 16 \\
Transient elevated IOP & 2 & 8 \\
Normal post operative patients & 14 & 56 \\
Late Complications & & \\
Cystoid macular edema & 2 & 20 \\
\hline
\end{tabular}

Table 2: Post-operative Visual outcomes.

\begin{tabular}{cc}
\hline Visual Acuity & No of Patients \\
\hline $6 / 6$ to $6 / 12$ & $3(12 \%)$ \\
$6 / 12$ to $6 / 18$ & $15(60 \%)$ \\
$6 / 18$ to $6 / 24$ & $4(16 \%)$ \\
$6 / 24$ to $6 / 60$ & $3(12 \%)$ \\
\hline
\end{tabular}




\section{DISCUSSION}

Suture-less scleral fixation is a unique advancement in complicated cataract surgeries. Different surgeons describe this technique in different ways. ${ }^{10}$ Our technique is very simple, needs less instrumentations and time saving. It is far superior technique than Scleral fixation with sutures. Sutures induce inflammation due to erosion. Other associated complications like suture breakage, subluxation and decentration of IOL are not seen in this technique.

Otha $\mathrm{T}$ reported dislocation of sutured IOL after 10 years in $99 \%$ of eyes. ${ }^{11}$ In our study, Marfan syndrome eyes with subluxated lenses were operated with this technique with good results. Similarly, Wilguckie et al performed sutureless scleral fixation and reported long-term stability and absence of suture associated decentrations. ${ }^{12}$ They described this technique as safe with long term stability and durability because haptics were incarcerated in the scleral tunnel giving it support for whole life. ${ }^{12}$ However, Gabor and Pavlidis' technique of inserting the IOL haptic into the scleral tunnel was not easy because of proximity of sclerotomy and scleral tunnel. ${ }^{13}$ In our study we made large and wide tunnels for easy haptic entry and positioning.

In our study, the results were encouraging regarding early and late complications of surgery and final visual acuity. Development of mild corneal edema in $5 \%$ eyes was due to second surgery after the first complicated procedure. Another complication was mild vitreous haemorrhage which occurred as a result of IOL and instrument manipulation during the procedure like pulling out IOL haptic through sclerotomies. It was resolved usually after one to two weeks. These were transient and manageable complications encountered in our study. Kim SS and Gabor and Totan Y reported same kind of complications in their studies. ${ }^{14,15,16}$ Haszcz et al considered it a long term stable IOL implantation technique. ${ }^{17}$ Visual results are also superior to anterior chamber IOL because this technique restores IOL to its probable anatomical position. ${ }^{17}$

Several new techniques including a trocar assisted, a suture-less $27 \mathrm{G}$ needle guarded, a Y-fixation intrascleral IOL implantation technique and Yamane technique are recently reported. ${ }^{18,19,20}$ Our technique gives us an opportunity to implant IOL with minimal manipulation and instrumentation.
Limitations of this case series is the small sample size and only two years follow up. Further multi-center trials with quasi experimental study designs are needed to prove its superiority.

\section{CONLUSION}

Sutureless Scleral fixation technique is simple, less time consuming and lifelong particularly in aphakic and syndromic children. It is without the problems related with sutures and IOL is close to its anatomical position. So, the chance of long term complications is less.

\section{Ethical Approval}

The study was approved by ethical review board of institution. (NMDC/993/20)

\section{Conflict of Interest}

Author declared no conflict of interest.

\section{REFERENCES}

1. McAllister AS, Hirst LW. Visual outcomes and complications of Scleral-fixation posterior chamber intraocular lenses. J Cataract Refract Surg. 2011; 37: 1263-1269.

2. Agarwal A, Kumar DA, Nair V. Cataract surgery in the setting of trauma. Curr Opin Ophthalmol. 2010 Jan;21(1):65-70.

3. Gabor SG, Pavlidis MM. Sutureless intrascleral posterior chamber intraocular lens fixation. J Cataract Refract Surgery, 2007; 33: 1851-1854.

4. Kim EJ, Brunin GM, Al-Mohtaseb ZN. Lens Placement in the Absence of Capsular Support: Scleralfixated Versus Iris-fixated IOL Versus ACIOL. Int Ophthalmol Clin. 2016 Summer;56(3):93-106.

5. Kumar DA, Agarwal A, Prakash D, Prakash G, Jacob S, Agarwal A. Glued intrascleral fixation of posterior chamber intraocular lens in children. Am J Ophthalmol. 20012; 153: 594-601.

6. Totan Y, Karadag R. Trocar-assisted Suture-less intrascleral posterior chamber foldable intra-ocular lens fixation. Eye, 2012; 26: 788-791.

7. Price MO, Price FW Jr, Werner L, Berlie C, Mamalis N. Late dislocation of scleral-sutured posterior chamber intraocular lenses. J Cataract Refract Surg. 2005 Jul;31(7):1320-6.

8. Scharioth GB, Prasad S, Gerogalas I, Tataru C, Pavlidis M. Intermediate results of Suture-less intrascleral posterior chamber intraocular lens fixation. J Cataract Refract Surg. 2010; 36: 254-259. 
9. Yamane S, Inoue M, Arakawa A, Kadonosono K. Sutureless 27 guage needle -guided intrascleral lens implantation with lamellar Scleral dissection. Ophthalmology, 2014; 121: 61-66.

10. Gabor SG, Pavlidis MM. Sutureless intrascleral posterior chamber intraocular lens fixation. J Cataract Refract Surg. 2007; 33: 1851-1854.

11. Otha T, Toshida H, Murakami A. Simplified and safe method of Sutureless intrascleral posterior chamber intraocular lens fixation: $\mathrm{Y}$ fixation technique. $\mathrm{J}$ Cataract Refract Surg. 2014; 40: 2-7.

12. Wilgucki JD, Wheatley HM, Feiner L, Ferrone MV, Prenner JL. One year outcomes of eyes treated with a suture-less Scleral fixation technique for intraocular lens placement or rescue. Rerina, 2015; 35: 1036-1040.

13. Gabor SG, Pavlidis MM. Suture-less intra-scleral posterior chamber intraocular lens fixation $\mathbf{J}$ Cataract Refract Surg. 2007; 33: 1851-1854.

14. Kim SS, Smiddy WE, Feuer W, Shi W. Management of dislocated intraocular lenses. Ophthalmology, 2008; 115 (10): 1699-1704.

15. Totan Y, Karadag R. Two techniques for Sutureless intrascleral posterior chamber IOL. J Refract Surg. 2013; 29 (2): 90-94.

Doi: 10.3928/1081597X-20130117-02.

16. Takayama K, Akimoto M, TaguchiH, Nakagawa S, Hiroi K. Transconjunctival sutureless intrascleral intraocular lens fixation using intrascleral tunnels guided with catheter and 30-guage needle," $\mathrm{Br} \mathrm{J}$ Ophthalmol. 2015; 99 (11): 1457-1459.
17. Haszcz D, Nowemiejska K, Oleszczuket A. Visual outcomes of posterior chamber intraocular lens intrascleral fixation in the setting of Postoperative and posttraumatic aphakia. BMC Ophthalmology, 2016; 16 (1): 50 .

18. Hu Z, Lin HS, Ye L, Lin Z, Chen T, Lin K, et al. Sutureless Intrascleral Haptic-Hook Lens Implantation Using 25-Gauge Trocars. J Ophthalmol. 2018; 9250425.

19. Por YM, Lavin MJ. Techniques of intraocular lens suspension in the absence of capsular/zonular support. Surv Ophthalmol. 2005; 50: 429469.

20. Yamane S, Ito A. Flanged fixation: Yamane technique and its application. Curr Opin Ophthalmol. 2021 Jan; 32 (1): 19-24.

\section{Authors' Designation and Contribution}

Muhammad Sajid Munir; Associate Professor: Concepts, Design.

Muhammad Ramzan; Professor: Literature search, Data acquisition.

Muhammad Arshad; Assistant Professor: Data Analysis, Statistical analysis.

Shahid Nazir; Associate Professor: Manuscript preparation, Manuscript editing.

Muhammad Rizwan Ullah; Associate Professor: Manuscript review. 